\title{
Neutralino Dark Matter in Minimal Supergravity: Direct Detection vs. Collider Searches
}

\author{
Howard Baer and Michal Brhlik \\ Department of Physics, Florida State University, Tallahassee, FL 32306 USA
}

(April 21, 2018)

\begin{abstract}
We calculate expected event rates for direct detection of relic neutralinos as a function of parameter space of the minimal supergravity model. Numerical results are presented for the specific case of a ${ }^{73} \mathrm{Ge}$ detector. We find significant detection rates $(R>0.01$ events $/ \mathrm{kg} /$ day $)$ in regions of parameter space most favored by constraints from $B \rightarrow X_{s} \gamma$ and the cosmological relic density of neutralinos. The detection rates are especially large in regions of large $\tan \beta$, where many conventional signals for supersymmetry at collider experiments are difficult to detect. If the parameter $\tan \beta$ is large, then there is a significant probability that the first direct evidence for supersymmetry could come from direct detection experiments, rather than from collider searches for sparticles.
\end{abstract}




\section{INTRODUCTION}

Luminous matter comprises less than $\Omega_{\text {lum }}=\rho / \rho_{c} \sim 0.01$ of the matter density of the universe [1,2], where $\rho_{c}=\frac{3 H_{0}^{2}}{8 \pi G_{N}}$ is the critical closure density of the universe, and $H=100 h \mathrm{~km} / \mathrm{sec} / \mathrm{Mpc}$ is the scaled Hubble constant, with $0.5 \lesssim h \lesssim 0.8$. In contrast, most inflationary cosmological models require $\rho=\rho_{c}$, i.e. a flat universe. The difference in these matter density values can be reconciled by hypothesizing the existence of dark (non-shining) matter (DM) in the universe. Experimental evidence for galactic dark matter comes from the enclosed mass versus velocity plots measured for clouds of neutral hydrogen rotating about galactic centers (galactic rotation curves), which imply $\Omega \geq 0.03-0.1$. An understanding of galactic clustering and galactic flows points towards even larger values of $\Omega \sim 0.2-1$, possibly in accord with inflationary cosmology. Calculations of Big Bang nucleosynthesis can only allow the baryonic contribution to the matter density of the universe to be $\Omega_{\text {baryonic }} \sim 0.01-0.1$, so that if $\rho=\rho_{c}$, the bulk of dark matter in the universe must be non-baryonic. Some candidates for non-baryonic dark matter from particle physics include neutrinos with $\mathrm{eV}$ scale masses (hot dark matter: HDM) and WIMPs (weakly interacting massive particles), such as axions or the lightest neutralino in supersymmetric (SUSY) models. Some compelling models of structure formation in the universe which take into account COBE measurements of the anisotropy in the cosmic microwave background actually prefer a "mixed dark matter" (MDM) universe, with $\sim 10 \%$ baryonic matter, $\sim 30 \%$ HDM, and $\sim 60 \%$ cold dark matter (CDM, comprised of WIMPs).

In this paper, we focus attention on the lightest SUSY particle, or LSP - usually the lightest neutralino - from supersymmetric models. At very early times after the Big Bang, neutralinos would have existed in thermal equilibrium with the primordial cosmic soup of particles and radiation. As the universe expanded and cooled, temperatures dropped so low that neutralinos could no longer be produced, although they could still annihilate away. Ultimately, the expansion rate of the universe exceeded the annihilation rate so that a relic density of neutralinos would be locked in. Numerous estimates of the neutralino relic density $\Omega h^{2}$ as a function of SUSY model parameter space have been made [3. [1]. Models that predict $\Omega h^{2}<0.025$ cannot even account for the dark matter needed for galactic rotation curves, while values of $\Omega h^{2}>1$ would yield a universe younger than 10 billion years old, in contradiction at least with the ages of the oldest stars found in globular clusters.

A consequence of the SUSY dark matter hypothesis is that a non-relativistic gas of neutralinos fills all space. To test this hypothesis, a number of direct detection experiments have been built or are under construction [5]. The general idea behind these experiments is that relic neutralinos (or other possible WIMPs) could scatter off the nuclei in some material, depositing typically tens of $\mathrm{keV}$ of energy. Some examples of how the thermal energy could be detected include: $i$.) via changes in resistance due to a slight temperature increase (bolometry), ii.) via a magnetic flux change due to a superconducting granule phase transition, or iii.) via ionization. The technical challenge is to build detectors that could pick out the relatively rare, low energy neutralino scattering events from backgrounds mainly due to cosmic rays and radioactivity in surrounding matter. Future detectors are aiming to reach a sensitivity of 0.1-0.01 events $/ \mathrm{kg} /$ day. In this way, the first evidence for SUSY might come from direct neutralino detection rather than from accelerator experiments.

Since the pioneering paper by Goodman and Witten [6], calculations of neutralino- 
nucleus scattering have seen continual improvements [7,8,8]. The first step involved in a neutralino-nucleus scattering calculation is to calculate the effective neutralino-quark and neutralino-gluon interactions. The neutralino-quark axial vector interaction leads in the non-relativistic limit to a neutralino-nucleon spin-spin interaction, which involves the measured quark spin content of the nucleon. To obtain the neutralino-nucleus scattering cross section, a convolution with nuclear spin form factors must be made. The neutralino-quark and neutralino-gluon interactions (via loop diagrams) can also resolve into scalar and tensor components. These interactions can be converted into an effective scalar neutralino-nucleon interaction involving quark and gluon parton distribution functions. A neutralino-nucleus scattering cross section can be obtained by convoluting with suitable scalar nuclear form factors. The final neutralino detection rate is obtained by multiplying by the local neutralino density (estimates are obtained from galaxy formation modeling), and appropriate functions involving the velocity distribution of relic neutralinos and the earth's velocity around the galactic center.

In this paper, we calculate event rates for direct detection of relic neutralinos left over from the Big Bang. For illustration, we present detailed calculations for a ${ }^{73} \mathrm{Ge}$ detector; a ${ }^{73} \mathrm{Ge}$ detector has a sizable nuclear spin content $J=\frac{9}{2}$, so that it would be sensitive to both spin and scalar neutralino-nucleus interactions [9]. Our goal is to compare the reach for supersymmetry by direct detection experiments with constraints on model parameter space from relic density calculations, collider search limits, and limits from $B \rightarrow X_{s} \gamma$ measurements. In addition, we will compare the reach of direct detection experiments with the previously calculated reach of collider facilities such as LEP2, the Fermilab Tevatron Main Injector (MI) upgrade and the CERN LHC $p p$ collider.

We work within the framework of the paradigm minimal supergravity (mSUGRA) model [10]. This model assumes the minimal supersymmetric standard model, or MSSM, is valid at all energy scales from $M_{\text {weak }}$ up to $M_{G U T} \simeq 2 \times 10^{16} \mathrm{GeV}$. The mSUGRA model could arise as the low energy limit of a supergravity theory, where supersymmetry is broken in the hidden sector of the model at energy scale $M \sim 10^{10} \mathrm{GeV}$. Supersymmetry breaking is communicated to the observable sector via gravitational interactions, leading to soft SUSY breaking mass terms of order the electroweak scale, $\tilde{m} \sim 100-1000 \mathrm{GeV}$. At the GUT scale (with the added assumption of an approximate global $U(n)$ symmetry for the mSUGRA Lagrangian), this leads to a common mass for all scalars $m_{0}$ and a common trilinear coupling $A_{0}$. Motivated by the apparent unification of gauge coupling constants, it is also assumed that all gaugino masses are unified to $m_{1 / 2}$ at $M_{G U T}$. The weak scale sparticle spectrum is derived from renormalization group (RG) running of the SUSY soft breaking parameters. Requiring radiative electroweak symmetry breaking allows the determination of the superpotential Higgsino mass squared $\mu^{2}$, and allows the expression of the soft SUSY breaking bilinear term $B$ in terms of $\tan \beta$, the ratio of vev's of the two Higgs fields. Thus, all sparticle masses and couplings are derived in terms of the parameter set

$$
m_{0}, m_{1 / 2}, A_{0}, \tan \beta, \text { and } \operatorname{sign}(\mu) .
$$

We adopt the mSUGRA spectrum calculation encoded into the event generator ISAJET 7.29 [11].

In Sec. 2, we present details of our calculation for neutralino scattering events off a ${ }^{73} \mathrm{Ge}$ detector. In Sec. 3 we present numerical results for event rates in the parameter space of the 
mSUGRA model, and compare to neutralino relic density contours. In Sec. 4, we compare our results to $B \rightarrow X_{s} \gamma$ constraints, and to collider search constraints. Finally, we compare the search capability of a ${ }^{73} \mathrm{Ge}$ dark matter detector to the reach of various future collider experiments for mSUGRA. We find usually that a DM detector attaining a sensitivity of 0.01 events $/ \mathrm{kg} /$ day will have a greater reach into mSUGRA parameter space than either the LEP2 or Tevatron upgrades, via their searches for sparticles. However, such dark matter detectors can only probe a fraction of the parameter space that gives rise to a reasonable relic density. A complete exploration of the cosmologically interesting mSUGRA parameter space will have to await the CERN LHC $p p$ collider, or an $e^{+} e^{-}$or $\mu^{+} \mu^{-}$collider operating at $\sqrt{s} \sim 1 \mathrm{TeV}$. In Sec. 5, we give a summary and some conclusions.

\section{CALCULATIONAL DETAILS}

\section{A. Dark Matter Detection: Theory}

The effective elastic scattering Lagrangian can generally be divided into two parts:

$$
\mathcal{L}_{\text {elastic }}^{\text {eff }}=\mathcal{L}_{\text {scalar }}^{\text {eff }}+\mathcal{L}_{\text {spin }}^{\text {eff }} .
$$

We examine first the scalar Lagrangian, which receives contributions from neutralino-quark interaction via squarks and Higgs bosons exchange, and from neutralino-gluon interactions at one-loop level involving quarks, squarks and Higgs bosons in the loop diagrams. On the parton level it is expressed at scale $Q$ (typically $\sim m_{\widetilde{Z}_{1}}$ ) as [8]

$$
\begin{aligned}
\mathcal{L}_{\text {scalar }}^{\text {eff }}= & f_{q} \overline{\widetilde{Z}}_{1} \widetilde{Z}_{1} \bar{q} q+g_{q}\left[-2 i \overline{\widetilde{Z}}_{1} \gamma_{\mu} \partial_{\nu} \widetilde{Z}_{1} \mathcal{Q}^{(2) \mu \nu}-\frac{1}{2} m_{q} m_{\widetilde{Z}_{1}} \overline{\widetilde{Z}}_{1} \widetilde{Z}_{1} \bar{q} q\right] \\
& +\alpha_{S}\left[-\left(B_{1 D}+B_{1 S}\right) \overline{\widetilde{Z}}_{1} \partial_{\mu} \partial_{\nu} \widetilde{Z}_{1}+B_{2 S} \overline{\widetilde{Z}}_{1}\left(i \gamma_{\mu} \partial_{\nu}+i \gamma_{\nu} \partial_{\mu}\right) \widetilde{Z}_{1}\right] \mathcal{G}^{(2) \mu \nu} \\
& +\alpha_{S} b \widetilde{\widetilde{Z}}_{1} \widetilde{Z}_{1} F_{\mu \nu}^{a} F^{a \mu \nu} .
\end{aligned}
$$

Here

$$
\begin{aligned}
\mathcal{Q}_{\mu \nu}^{(2)} & =\frac{i}{2}\left(\bar{q} \gamma_{\mu} \partial_{\nu} q+\bar{q} \gamma_{\nu} \partial_{\mu} q-\frac{1}{2} g_{\mu \nu} \bar{q} \not \partial q\right) \\
\mathcal{G}_{\mu \nu}^{(2)} & =F_{\mu \rho}^{a} F_{\nu}^{a \rho}+\frac{1}{4} g_{\mu \nu} F_{\rho \sigma}^{a} F^{a \rho \sigma}
\end{aligned}
$$

are traceless twist-2 quark and gluon operators, and for the sake of brevity the effective couplings are given in the Appendix. Using nucleonic matrix elements

$$
\begin{aligned}
\left\langle N\left|\mathcal{Q}_{\mu \nu}^{(2)}\left(q^{2} \rightarrow 0\right)\right| N\right\rangle & =\frac{1}{m_{N}}\left(p_{\mu} p_{\nu}-\frac{1}{4} m_{N}^{2} g_{\mu \nu}\right) \int_{0}^{1} x\left[q_{N}(x, Q)+\bar{q}_{N}(x, Q)\right] d x \\
\left\langle N\left|\mathcal{G}_{\mu \nu}^{(2)}\left(q^{2} \rightarrow 0\right)\right| N\right\rangle & =\frac{1}{m_{N}}\left(p_{\mu} p_{\nu}-\frac{1}{4} m_{N}^{2} g_{\mu \nu}\right) \int_{0}^{1} x g_{N}(x, Q) d x
\end{aligned}
$$

(here, $q^{2}$ is the momentum transfer squared, and $p_{\mu}$ is the nucleon four-momentum) and introducing the parton distribution functions $q_{N}, \bar{q}_{N}$ and $g_{N}$, and using

$$
\left\langle N\left|F_{\rho \sigma}^{a} F^{a \rho \sigma}\left(q^{2} \rightarrow 0\right)\right| N\right\rangle=\frac{8 \pi}{9 \alpha_{S}} m_{N} f_{T G}^{(N)},
$$




$$
\left\langle N\left|m_{q} \bar{q} q\right| N\right\rangle=m_{N} f_{T q}^{(N)}
$$

it is possible to convert Lagrangian (2.15) into an effective neutralino-nucleon Lagrangian

$$
\mathcal{L}_{\text {scalar }}^{\text {eff }}=f_{p} \overline{\widetilde{Z}}_{1} \widetilde{Z}_{1} \bar{\Psi}_{p} \Psi_{p}+f_{n} \overline{\widetilde{Z}}_{1} \widetilde{Z}_{1} \bar{\Psi}_{n} \Psi_{n}
$$

Evaluation of $f_{N}$ for $N=p, n$ yields

$$
\begin{aligned}
\frac{f_{N}}{m_{N}}= & \sum_{u, d, s} \frac{f_{T q}^{(N)}}{m_{q}}\left[f_{q}^{(\tilde{q})}+f_{q}^{(H)}-\frac{1}{2} m_{q} m_{\widetilde{Z}_{1}} g_{q}\right]+\frac{2}{27} f_{T G}^{(N)} \sum_{c, b, t} \frac{f_{q}^{(H)}}{m_{q}} \\
& -\frac{3}{2} m_{\widetilde{Z}_{1}} \sum_{\substack{u, d, s \\
c, b}} g_{q}(Q)\left(q_{N}(2, Q)+\bar{q}_{N}(2, Q)\right)-\frac{8 \pi}{9} b f_{T G}^{(N)} \\
& +\frac{3}{2} \alpha_{S} m_{\widetilde{Z}_{1}}\left[B_{2 S}+\frac{1}{2} m_{\widetilde{Z}_{1}}\left(B_{1 D}+B_{1 S}\right)\right] g_{N}(2, Q),
\end{aligned}
$$

where the various coupling constants are given in the Appendix. Here we have used the general definition of an $n$-th integral moment $f(n, Q)=\int_{0}^{1} x^{n-1} f(x, Q) d x$ and applied it to the parton distribution functions. The differential cross section for a neutralino scattering off a nucleus $X_{Z}^{A}$ with mass $m_{A}$ is then expressed as

$$
\frac{d \sigma^{\text {scalar }}}{d|\vec{q}|^{2}}=\frac{1}{\pi v^{2}}\left[Z f_{p}+(A-Z) f_{n}\right]^{2} F^{2}\left(Q_{r}\right)
$$

where $\vec{q}=\frac{m_{A} m_{\widetilde{z}_{1}}}{m_{A}+m_{\widetilde{Z}_{1}}} \vec{v}$ is the transfered momentum, $Q_{r}=\frac{|\vec{q}|^{2}}{2 m_{A}}$ and $F^{2}\left(Q_{r}\right)$ is the scalar nuclear form factor.

Interaction between the neutralino and quark spins is described by a parton-level Lagrangian [2]

$$
\mathcal{L}_{\text {spin }}^{\text {eff }}=d_{q} \overline{\widetilde{Z}}_{1} \gamma^{\mu} \gamma_{5} \widetilde{Z}_{1} \bar{q} \gamma_{\mu} \gamma_{5} q
$$

which translates with the help of nucleonic spin matrix elements

$$
<N\left|\bar{q} \gamma_{\mu} \gamma_{5} q\right| N>=2 s_{\mu} \Delta q^{(N)}
$$

into

$$
\mathcal{L}_{\text {spin }}^{e f f}=2 \sqrt{2}\left(a_{p} \overline{\widetilde{Z}}_{1} \gamma^{\mu} \gamma_{5} \widetilde{Z}_{1} \bar{\Psi}_{p} s_{\mu} \Psi_{p}+a_{n} \overline{\widetilde{Z}}_{1} \gamma^{\mu} \gamma_{5} \widetilde{Z}_{1} \bar{\Psi}_{n} s_{\mu} \Psi_{n}\right)
$$

explicitly involving the nucleon spin vectors $s_{\mu}$. Coefficients

$$
a_{p}=\frac{1}{\sqrt{2}} \sum_{u, d, s} d_{q} \Delta q^{(p)}, \quad a_{n}=\frac{1}{\sqrt{2}} \sum_{u, d, s} d_{q} \Delta q^{(n)}
$$

depend on experimental values of $\Delta q^{(N)}$, which are affected by significant uncertainties which lead to variations in the cross section. More details on the couplings are again given in the Appendix. For a nucleus with total angular momentum $J$, the spin interaction differential cross section takes the form 


$$
\frac{d \sigma^{\text {spin }}}{d|\vec{q}|^{2}}=\frac{8}{\pi v^{2}} \Lambda^{2} J(J+1) \frac{S(|\vec{q}|)}{S(0)}
$$

where $\frac{S(|\vec{q}|)}{S(0)}$ is the nuclear spin form factor normalized to 1 for pointlike particles, and $\Lambda=\frac{1}{J}\left[a_{p}\left\langle S_{p}\right\rangle+a_{n}\left\langle S_{n}\right\rangle\right]$. The quantities $\left\langle S_{p}\right\rangle$ and $\left\langle S_{n}\right\rangle$ represent the expectation value of the proton (neutron) group spin content in the nucleus.

Putting both scalar and spin interaction contributions together and convoluting them with the local neutralino flux (which depends on the local relic density $\rho_{\widetilde{Z}_{1}}$ ), the differential detection rate is calculated to be

$$
\begin{aligned}
\frac{d R}{d Q_{r}}=\frac{4}{\sqrt{\pi^{3}}} & \frac{\rho_{\widetilde{Z}_{1}}}{m_{\widetilde{Z}_{1}} v_{0}} T\left(Q_{r}\right)\left\{\left[Z f_{p}+(A-Z) f_{n}\right]^{2} F^{2}\left(Q_{r}\right)\right. \\
& \left.+8 \Lambda^{2} J(J+1) \frac{S(|\vec{q}|)}{S(0)}\right\}
\end{aligned}
$$

where $v_{0} \sim 220 \mathrm{~km} / \mathrm{s}$ is the circular speed of the Sun around the center of our galaxy and

$$
T\left(Q_{r}\right)=\frac{\sqrt{\pi} v_{0}}{2} \int_{v_{\min }}^{\infty} \frac{f_{\widetilde{Z}_{1}}}{v} d v
$$

integrates over the neutralino velocity distribution.

\section{B. Detection Rates in Germanium Detectors}

In order to obtain a quantitative estimate of neutralino detection rates in the mSUGRA framework which could be compared to phenomenological constraints and collider reaches, we have evaluated the rate for the case of a ${ }^{73} \mathrm{Ge}$ detector. In our calculations, we have taken the neutralino density to be the local relic density consistent with galactic formation models including varying baryonic mass fraction in the galaxy [12]. In the simplest Gaussian model taking into account the motion of the Sun and Earth, the integrated velocity distribution can be written as [2]

$$
T\left(Q_{r}\right)=\frac{\sqrt{\pi} v_{0}}{4 v_{e}}\left[\operatorname{Erf}\left(\frac{v_{\min }+v_{e}}{v_{0}}\right)-\operatorname{Erf}\left(\frac{v_{\min }-v_{e}}{v_{0}}\right)\right]
$$

with $v_{\text {min }}=\sqrt{\frac{Q_{r}\left(m_{\widetilde{Z}_{1}}+m_{A}\right)^{2}}{2 m_{\widetilde{Z}_{1}}^{2} m_{A}}}$, and where the Earth velocity $v_{e}$ is given by

$$
v_{e}=v_{0}\left[1.05+0.07 \cos \left(\frac{2 \pi\left(t-t_{p}\right)}{1 y r}\right)\right]
$$

with $t_{p} \simeq$ June 2 . The general nuclear properties needed are the ${ }^{73}$ Ge mass, $m_{G e}=67.93$ $\mathrm{GeV}$, and its total spin $J=\frac{9}{2}$.

To determine the scalar contribution, it is necessary to compute the parton distribution integrals at a scale defined by the average squark mass and neutralino mass $Q^{2} \simeq\left(m_{\widetilde{q}}^{2}-m_{\widetilde{Z}_{1}}^{2}\right)$. We employ the CTEQ3L parton distribution function parametrization [13] for numerical 
calculation. The most recent values of the matrix element coefficients $f_{T q}^{(N)}$ and $f_{T G}^{(N)}=$ $1-\sum_{q} f_{T q}^{(N)}$ were compiled by [2] giving $f_{T u}^{(p)}=0.019, f_{T d}^{(p)}=0.041, f_{T u}^{(n)}=0.023, f_{T d}^{(n)}=0.034$ and $f_{T s}^{(p)}=f_{T s}^{(n)}=0.14$. We adopt the Saxon-Woods scalar form factor suggested in [14]

$$
F\left(Q_{r}\right)=\frac{3 j_{1}\left(q R_{0}\right)}{q R_{1}} e^{-\frac{1}{2}(q s)^{2}}
$$

where $R_{1}=\sqrt{R^{2}-5 s^{2}}, R=A^{\frac{1}{3}} \times 1.2 \mathrm{fm}, j_{1}$ is a spherical Bessel function and $s=1 \mathrm{fm}$.

In the case of the spin interaction, the spin analogues of the parton distibution functions are much less well known, and we take [15] $\Delta u_{p}=\Delta d_{n}=0.78, \Delta d_{p}=\Delta u_{n}=-0.5$ and $\Delta s_{p}=\Delta s_{n}=-0.16$. Theoretical predictions for the spin content of the two nucleon groups in the nucleus and the spin form factor are very model dependent. To make a consistent choice, we follow [16], where for ${ }^{73} \mathrm{Ge}\left\langle S_{p}\right\rangle=0.03,\left\langle S_{n}\right\rangle=0.378$ and the form factor is given as

$$
S(q)=\left(a_{p}+a_{n}\right)^{2} S_{00}(q)+\left(a_{p}-a_{n}\right)^{2} S_{11}(q)+\left(a_{p}^{2}-a_{n}^{2}\right) S_{01}(q)
$$

and the $S_{i j}$ individual form factors are evaluated as polynomial fits to data.

As a final step in the calculation, the differential rate $\frac{d R}{d Q_{r}}$ must be integrated over $Q_{r}$ ranging typically from $\sim 0$ to less than $100 \mathrm{keV}$. We will show the rate $R$ in events $/ \mathrm{kg} /$ day for a particular choice of the assumed local neutralino density $\rho_{\widetilde{Z_{1}}}=5 \times 10^{-25} \mathrm{~g} \mathrm{~cm}^{-3}$ [12]. The standard lore is that the local halo density uncertainty should be roughly a factor of two; a more accurate prediction based on galaxy formation models would be needed to reduce it.

\section{NEUTRALINO DETECTION RATES IN MSUGRA PARAMETER SPACE}

We show our first numerical results for direct detection of neutralinos in Fig. 1, where we plot contours of scattering events $/ \mathrm{kg} /$ day for a ${ }^{73} \mathrm{Ge}$ detector as a function of mSUGRA parameters $m_{0}$ vs. $m_{1 / 2}$ with $A_{0}=0, \tan \beta=2$ and $\left.a\right) \mu<0$ and $\left.b\right) \mu>0$. The region labelled TH is excluded by theoretical consideration: either the LSP is charged or colored (not the lightest neutralino), or radiative electroweak symmetry breaking is not properly attained. The region labelled EX is excluded by collider searches for SUSY particles. By far the strongest of these for mSUGRA is the recent limit from LEP2 that $m_{\widetilde{W}_{1}}>85 \mathrm{GeV}$ [17]. We also show the contour of constant $\Omega h^{2}=1$ (solid); beyond this contour, mSUGRA parameter space points lead to universe with age less than 10 billion years. In addition, the dot-dashed contours correspond to $\Omega h^{2}=0.15$ and 0.4 ; the region between these contours is favored by MDM cosmological models.

We find in frame $a$ ) that the direct dark matter detection rates are uniformly low throughout the parameter space shown; for all points sampled, we found $R<0.01 / \mathrm{kg} /$ day, which is less than the goal for such detectors at least in the near-term future. In frame $b$ ), however, for $\mu>0$, we find larger rates for dark matter detection, with a significant fraction of parameter space with $m_{1 / 2}<200 \mathrm{GeV}$ accessible to dark matter detectors achieving a sensitivity of $R \gtrsim 0.01 / \mathrm{kg} /$ day. Note that the $R \gtrsim 0.01 / \mathrm{kg} /$ day region overlaps with the lower portion of the region favored by a MDM universe.

In Fig. 2, we show similar results, except now we take $\tan \beta=10$. In Fig. 2, the region below the dotted contour is where $\Omega h^{2}<0.025$ - too small to account for the galactic 
rotation curves. In frame $a$ ), we find considerably larger detection rates than for Fig. $1 a$, with $R$ reaching values $\sim 0.1$ in the lower-left. However, in this region, $\Omega h^{2}<0.025$ so that the highest detection rates exist in an uninteresting region of relic density. DM detectors with sensitivity $R \gtrsim 0.01 / \mathrm{kg} /$ day can probe just the lower portion of the MDM cosmologically favored region. Likewise, in frame $b$ ), event rates are larger as well than in Fig. 1b, with the entire region shown below $m_{1 / 2} \simeq 200 \mathrm{GeV}$ accessible to DM detectors able to achieve a counting rate of $R=0.01 / \mathrm{kg} /$ day.

Fig. 3 is similar in construction to Figs. 1 and 2 , but with $\tan \beta=35$. Note, however, the expanded scale relative to Figs. 1 and 2. The first peculiarity of note is that the TH region has expanded considerably. This is due to the magnitude of the $\tau$ Yukawa coupling at large $\tan \beta$, which drives the $\tilde{\tau}_{1}$ mass to lower values than corresponding sleptons from the first two generations. The expanded TH region in the upper left is thus where $m_{\tilde{\tau}_{1}}<m_{\widetilde{Z}}$, so that the $\tilde{\tau}_{1}$ is the LSP instead of the lightest neutralino.

In Fig. $3 a$ and $b$, we see that the regions of cosmologically interesting relic density $\Omega h^{2}$ are much larger than for the low $\tan \beta$ cases. In this case, the enhanced Higgs coupling to $b \bar{b}$ and $\tau \bar{\tau}$ at large $\tan \beta$ gives rise to a very broad resonance structure so that $s$-channel annihilation of neutralinos is possible over a very large region of parameter space. Note in particular that no upper limit on SUSY particle masses is evident in these plots from the $\Omega h^{2}<1$ constraint.

We find in Fig. 3 that the dark matter detection rate $R$ has grown even larger for low $m_{1 / 2}$ values, relative to Figs. 1 and 2 , so that $R$ exceeds 1 event $/ \mathrm{kg} /$ day in the lower left! In this region, however, $\Omega h^{2}<0.025$ so that again the largest detection rates are in a cosmologically uninteresting region. We see that $R$ can exceed values of $0.01 / \mathrm{kg} / \mathrm{day}$ for $m_{1 / 2}$ as high as $300 \mathrm{GeV}$, which corresponds to a reach in $m_{\tilde{g}}$ of $\sim 750 \mathrm{GeV}$ !

The large increase in DM detection rate for large $\tan \beta$ has been noted previously by Drees and Nojiri [8]. This is a fortuitous result: the region of parameter space where DM detection is easiest is precisely the region of parameter space where collider detection of SUSY particles is most difficult (this will be discussed in more detail in Sec. 4). We show the explicit variation in $R$ with $\tan \beta$ for the mSUGRA point $m_{0}, m_{1 / 2}, A_{0}=150,200,0 \mathrm{GeV}$ in Fig. 4. Here we see the event rate increasing by more than one (two) orders of magnitude with $\tan \beta$ for $\mu>0(\mu<0)$. Fig. 4 has some resemblance to Fig. 3 of the second paper of Ref. [ 8 ]; in our case (for a ${ }^{73} \mathrm{Ge}$ detector instead of a spin- $0{ }^{76} \mathrm{Ge}$ detector), the large spin-spin interaction causes the main difference between the plots.

For ${ }^{73} \mathrm{Ge}$, the spin coupling is large, and can cause the axial-vector interaction to exceed the scalar interaction even for nuclei as heavy as Ge. This is shown in Fig. 5, where we plot the ratio $R_{\text {spin }} / R_{\text {scalar }}$ for the same mSUGRA point as in Fig. 4. Here, we see that the spin interaction actually dominates for $\tan \beta \sim 6$, for $\mu<0$. This is contrary to naive expectations that the spin interaction is always sub-dominant for nuclei with $A \gtrsim 20$.

In Fig. 6, we show an example of the variation in dark matter detection rate versus variation in the trilinear soft-breaking term $A_{0}$, for $m_{0}, m_{1 / 2}=150,200 \mathrm{GeV}$, and $\tan \beta=10$. From this plot, we see that the DM detection rate can vary with $A_{0}$ by an order of magnitude; this sort of variation must be taken into account if dark matter detection experiments ever try to obtain limits on mSUGRA parameter space.

Finally, we note that a seasonal variation in the DM detection rate is expected [2]. This is caused by accounting for the earth's velocity about the sun, while at the same time 
accounting for the sun's velocity about the galactic center. In Fig. 7, we plot the DM detection rate as a function of month of the year (beginning with Jan. 1). The maximum detection rate occurs around June 2, although the seasonal variation amounts to less than a per cent, so that very high counting rates would be necessary to detect this. We note as well that if DM detectors are sensitive to the direction of collision products, these should also depend on the time of day and season of the year.

\section{COMPARISON WITH SUSY SEARCHES AT COLLIDERS}

In this section, we compare our results for direct detection of neutralino DM with constraints from $B \rightarrow X_{s} \gamma$ searches, and with expectations for various collider searches. In these comparisons, we are restricted to values of $\tan \beta<10$, for which detailed collider and $B \rightarrow X_{s} \gamma$ calculations are available. Only recently has the event generator ISAJET been upgraded to handle large $\tan \beta$ cases, so detailed studies for collider expectations still need to be made [18].

The rare decay $B \rightarrow X_{s} \gamma$ has been shown to yield rather strong constraints on supersymmetric models, due to loop amplitudes containing charginos, neutralino, gluinos, squarks and charged Higgs bosons [19]. In a recent paper [20], a QCD-improved calculation of the $B \rightarrow X_{s} \gamma$ branching fraction has been made for the mSUGRA model. The sensitivity to variations in the QCD renormalization scale has been reduced considerably compared to previous results. In this paper, only chargino, charged Higgs and $W$-boson loops have been included, which is appropriate for small to moderate values of $\tan \beta$. Comparison of calculated branching fractions to recent results from the CLEO experiment has resulted in identification of regions of mSUGRA model parameter space which are excluded at $95 \%$ CL. For large $\tan \beta, \tilde{g}$ and $\widetilde{Z}_{i}$ loops will also be relevant (see Borzumati, Drees and Nojiri, Ref. [19]). In this case, the derived constraints will depend sensitively upon details of the assumed structure of high scale squark mass matrices, so that the implications will be much more model dependent.

In Fig. 9, we again show the $m_{0}$ vs. $m_{1 / 2}$ plane for $\tan \beta=2$. This time, we show in Fig. $9 a$ the region excluded by the $95 \%$ CL CLEO result compared to mSUGRA model calculations. We exclude parameter space points where the $B \rightarrow X_{s} \gamma$ branching ratio falls outside the CLEO 95\% CL limits for all choices of renormalization scale $\frac{m_{b}}{2}<Q<2 m_{b}$. The excluded region is in the lower left of frame $a$ ), where the dark matter detection rate is largest, although still smaller than $R=0.01 / \mathrm{kg} /$ day. In this frame, we also show the region that can be searched by the LEP2 $e^{+} e^{-}$collider operating at $\sqrt{s}=190 \mathrm{GeV}$, and accumulating $\sim 500 \mathrm{pb}^{-1}$ of integrated luminosity (dotted curve). The left-side bulge in this contour is where LEP2 is sensitive to selectron searches, while below the right-hand side, which asymptotically approaches $m_{1 / 2} \simeq 100$, is where LEP2 is sensitive to chargino pair searches [21]. We also note that LEP2 is sensitive to almost the entire plane shown via the $e^{+} e^{-} \rightarrow Z h$ search channel; in this case, however, it will be difficult to tell the light SUSY Higgs $h$ from the SM Higgs boson. Finally, we also show the dashed contour, which is the reach of the Tevatron Main Injector (MI: $\sqrt{s}=2 \mathrm{TeV}$; integrated luminosity $=2 \mathrm{fb}^{-1}$ ). This latter curve mainly results from regions where the clean trilepton signal from $\widetilde{W}_{1} \widetilde{Z}_{2} \rightarrow 3 \ell$ is observable above SM backgrounds [22]. By comparing all the contours of Fig. $9 a$, we note that the low $m_{1 / 2}$ region of the MDM-favored region is excluded by $B \rightarrow X_{s} \gamma$, which also 
excludes much of the region open to SUSY discovery by LEP2 and Tevatron MI searches. If mSUGRA is correct, with $\tan \beta=2$ and $\mu<0$, then LEP2 may well discover the light Higgs scalar $h$, but probably there will be no direct detection of SUSY DM and SUSY discovery will have to wait for the CERN LHC $p p$ collider, which has the ability to explore the entire SUSY parameter space with relatively low luminosity [23].

In Fig. $9 b$, none of the $m_{0}$ vs. $m_{1 / 2}$ plane is excluded by $B \rightarrow X_{s} \gamma$. The reach of LEP2 is almost always below the MDM-favored region. However, both the Tevatron MI as well as DM detectors sensitive to $R \simeq 0.01 / \mathrm{kg} /$ day can explore the lower limits of the MDM region. In this case, the MI has somewhat of a better reach for SUSY than direct DM detection experiments, while LEP2 would stand again a good chance to find the light Higgs scalar $h$ 21 .

In Fig. $10 a$, we show the plane for $\tan \beta=10$ and $\mu<0$. The outstanding feature here is that $B \rightarrow X_{s} \gamma$ data exclude almost the whole plane below $m_{1 / 2} \sim 350 \mathrm{GeV}$ (corresponding to $m_{\tilde{g}} \lesssim 900 \mathrm{GeV}$ ), including essentially all of the MDM-favored region [20]! If mSUGRA exists in this parameter plane, then direct DM detection experiments, LEP2 and Tevatron MI will find no evidence of SUSY, and SUSY discovery will have to await the LHC (although $B \rightarrow X_{s} \gamma$ experiments may have a strong hint of new physics).

Fig. $10 b$ shows the $m_{0}$ vs. $m_{1 / 2}$ plane for $\tan \beta=10$ and $\mu>0$. In this case, none of the plane shown is excluded by $B \rightarrow X_{s} \gamma$. In fact, the mSUGRA region around $m_{1 / 2} \simeq 200$ GeV gives a better match to CLEO data than does the SM! For this case, the reach of LEP2 is below the MDM region, and in addition the Higgs scalar $h$ is too heavy for detection at LEP2. The Tevatron MI can explore a portion of the MDM favored region. However, a DM detector with sensitivity $R \simeq 0.01 / \mathrm{kg} /$ day will be sensitive to the additional region with $m_{1 / 2} \lesssim 200 \mathrm{GeV}$, and $m_{0} \gtrsim 150 \mathrm{GeV}$, which is inaccessible to Tevatron or LEP2 searches. This region includes the entire MDM-favored $s$-channel annihilation corridor for which $m_{1 / 2} \sim 180 \mathrm{GeV}$. This latter region is also favored by the $B \rightarrow X_{s} \gamma$ measurement 20].

Finally, we coment upon the large $\tan \beta$ region, for which detailed collider studies and $B \rightarrow X_{s} \gamma$ calculations have yet to be made. Drees and Nojiri pointed out that as $\tan \beta$ increase, the Higgs pseudoscalar mass $m_{A}$ decreases significantly, so that over much of parameter space the neutralino relic density decreases, mainly due to $s$-channel $\widetilde{Z} \widetilde{Z} \rightarrow A, H \rightarrow b \bar{b}$ annihilation reactions [3]. We have verified this with the relic density contours presented in Fig. 3. In a recent paper [18], detailed calculations of sparticle masses, production and decay processes at large $\tan \beta$ were reported. It was noted that for large $\tan \beta$, the $\widetilde{W}_{1}$ and $\widetilde{Z}_{2}$ branching ratios to $\tau$ leptons and $b$-quarks increases due to Yukawa coupling effects, which leads to a diminution of the corresponding branchings to easily detectable $e$ and $\mu$ states. This generally ought to make SUSY detection much more difficult for the Tevatron MI than corresponding mSUGRA points with low $\tan \beta$. However, we note from Fig. 3 that the large $\tan \beta$ region is precisely where DM detection rates can be largest. Hence, if $\tan \beta$ is large, it is possible that the first evidence for SUSY might come from direct dark matter detection, rather than from LEP2 or MI searches. It is expected that the CERN LHC $p p$ collider will still be able to cover the entire mSUGRA parameter space even if $\tan \beta$ is large, at least via multijet $+E_{T}$ searches [18]. 


\section{SUMMARY AND CONCLUSIONS}

In this paper, we have presented expected event rates for direct detection of neutralinos by cryogenic dark matter detectors. To be specific, we chose to perform calculations for a ${ }^{73} \mathrm{Ge}$ detector. Many other choices of materials are possible, and analogous calculations can be made by using different nuclear $A, Z$ and $J$ values, and by using different nuclear form factors. Our main results were presented in Figs. 1-3 as functions of mSUGRA parameter space. When interpreting these results, the intrinsic uncertainties in the calculations should be kept in mind. Roughly, we expect a factor of $\sim 2$ uncertainty from each of $i$.) calculations in leading-log QCD, ii.) uncertainty in knowledge of the local dark matter relic density and iii.) uncertainty in nuclear form factors and in quark contributions to nucleon spin. Simplistically adding these in quadature would imply our results for event rates are only reliable to a factor $3-4$. In addition, variation of the parameter $A_{0}$ can cause changes in the DM detection rates by up to an order of magnitude (see Fig. 6).

One of our main goals in this paper was to compare the reach for supersymmetry by dark matter detectors against the reach for SUSY via collider experiments and rare decay searches. A dark matter detector reaching a sensitivity of $R \sim 0.01 / \mathrm{kg} /$ day usually would have a better reach in mSUGRA parameter space than LEP2 would for SUSY particles (but not if one includes LEP2 sensitivity to Higgs bosons). Dark matter detectors can be comparable to the Tevatron MI in terms of reach for mSUGRA for low values of $\tan \beta$. However, for large $\tan \beta$, dark matter detectors have an increased event rate, whereas the reach of the Tevatron MI will likely diminish relative to capabilities at modest values of $\tan \beta$. For the large $\tan \beta$ case, the first direct evidence for SUSY may well come from direct detection experiments. We note that even if dark matter detectors only achieve a sensitivity of $0.1 / \mathrm{kg} /$ day, they would still have a substantial reach for SUSY in the large $\tan \beta$ region. The relative capabilities at low and high $\tan \beta$ underscores another facet of complementarity between collider search experiments, and direct detection of dark matter. Obviously, if one detects SUSY at collider experiments, it would still be fascinating to verify the existence and properties of neutralino dark matter.

\section{ACKNOWLEDGMENTS}

We thank M. Drees and X. Tata for comments on the manuscript. This research was supported in part by the U. S. Department of Energy under grant number DE-FG-0587ER40319. 


\section{APPENDIX A: EFFECTIVE COUPLINGS FOR NEUTRALINO-NUCLEON SCATTERING}

In this Appendix we summarize effective couplings entering the scalar and spin Lagrangian for neutralino scattering on nucleons, and express them in terms of the MSSM coupling constants and masses. Most of the time we follow reference [8].

Both interactions are mediated by squarks, Higgs bosons or the $\mathrm{Z}^{0}$ boson connecting either directly or through a loop the neutralino lines of propagation with quark (gluon) lines in the nucleon. The lightest neutralino-quark-squark Lagrangian reads

$$
\mathcal{L}_{\widetilde{Z}_{\ell} q \widetilde{q}}=\sum_{i=1,2} \overline{\widetilde{Z}}_{\ell}\left(a_{\widetilde{q}_{i}}+b_{\widetilde{q}_{i}} \gamma_{5}\right) q \widetilde{q}_{i}+\text { h.c. }
$$

and the $a$ and $b$ coefficients take the form

$$
\begin{aligned}
a_{\tilde{q}_{i}} & =\frac{1}{\sqrt{2}}\left\{-M_{i 1}\left[e e_{q} N_{\ell 1}^{\prime}+\frac{g}{\cos \theta_{W}} N_{\ell 2}^{\prime}\left(T_{3 q}-e_{q} \sin ^{2} \theta_{W}\right)+\frac{g m_{q}}{2 m_{W}} N_{\ell x}\right]\right. \\
& \left.+M_{i 2}\left[e e_{q} N_{\ell 1}^{\prime}-\frac{g}{\cos \theta_{W}} N_{\ell 2}^{\prime} e_{q} \sin ^{2} \theta_{W}-\frac{g m_{q}}{2 m_{W}} N_{\ell x}\right]\right\} \\
b_{\tilde{q}_{i}} & =\frac{1}{\sqrt{2}}\left\{-M_{i 1}\left[e e_{q} N_{\ell 1}^{\prime}+\frac{g}{\cos \theta_{W}} N_{\ell 2}^{\prime}\left(T_{3 q}-e_{q} \sin ^{2} \theta_{W}\right)-\frac{g m_{q}}{2 m_{W}} N_{\ell x}\right]\right. \\
& \left.+M_{i 2}\left[-e e_{q} N_{\ell 1}^{\prime}+\frac{g}{\cos \theta_{W}} N_{\ell 2}^{\prime} e_{q} \sin ^{2} \theta_{W}-\frac{g m_{q}}{2 m_{W}} N_{\ell x}\right]\right\}
\end{aligned}
$$

where

$$
\left(\begin{array}{c}
\widetilde{q}_{1} \\
\widetilde{q}_{2}
\end{array}\right)=\left(\begin{array}{ll}
M_{11} & M_{12} \\
M_{21} & M_{22}
\end{array}\right),\left(\begin{array}{c}
\widetilde{q}_{L} \\
\widetilde{q}_{R}
\end{array}\right)=\left(\begin{array}{cc}
\cos \theta_{q} & \sin \theta_{q} \\
-\sin \theta_{q} & \cos \theta_{q}
\end{array}\right)\left(\begin{array}{c}
\widetilde{q}_{L} \\
\widetilde{q}_{R}
\end{array}\right)
$$

and $x=3(4)$ for a down (up) type quark. The $4 \times 4 N$ matrix diagonalizes the neutralino mass matrix and

$$
\left(\begin{array}{c}
N_{j 1}^{\prime} \\
N_{j 2}^{\prime}
\end{array}\right)=\left(\begin{array}{cc}
\cos \theta_{W} & \sin \theta_{W} \\
-\sin \theta_{W} & \cos \theta_{W}
\end{array}\right)\left(\begin{array}{c}
N_{j 1} \\
N_{j 2}
\end{array}\right) .
$$

The $f_{q}$ coupling in $(2.15)$ can be split into two parts

$$
f_{q}=f_{q}^{(\widetilde{q})}+f_{q}^{(H)}
$$

where the squark part is

$$
f_{q}^{(\widetilde{q})}=-\frac{1}{4} \sum_{i=1,2} \frac{a_{\widetilde{q}_{i}}^{2}-b_{\widetilde{q}_{i}}^{2}}{m_{\widetilde{q}_{i}}^{2}-\left(m_{\widetilde{Z}_{1}}+m_{q}\right)^{2}},
$$

and the Higgs exchange part is

$$
f_{q}^{(H)}=m_{q} \sum_{j=1,2} \frac{c_{\widetilde{Z}}^{(j)} c_{q}^{(j)}}{m_{H_{j}}^{2}}
$$


Mixing in the Higgs sector results in

$$
c_{\widetilde{Z}}^{(1)}=\frac{1}{2}\left(g N_{\ell 2}-g^{\prime} N_{\ell 1}\right)\left(N_{\ell 3} \sin \alpha+N_{\ell 4} \cos \alpha\right)
$$

for the lighter CP-even Higgs and

$$
c_{\widetilde{Z}}^{(2)}=\frac{1}{2}\left(g N_{\ell 2}-g^{\prime} N_{\ell 1}\right)\left(N_{\ell 4} \sin \alpha-N_{\ell 3} \cos \alpha\right)
$$

for the heavier Higgs, where $\alpha$ is the Higgs mixing angle. The quark coefficients are evaluated as

$$
c_{q}^{(i)}=\frac{g}{2 m_{W}} r_{q}^{(i)}
$$

with

$$
r_{u}^{(1)}=-\frac{\sin \alpha}{\sin \beta}, \quad r_{u}^{(2)}=-\frac{\cos \alpha}{\sin \beta}
$$

for the up type quarks and

$$
r_{d}^{(1)}=-\frac{\cos \alpha}{\cos \beta}, \quad r_{d}^{(2)}=\frac{\sin \alpha}{\cos \beta}
$$

for the down type quarks. The quark tensor contribution coupling in (2.15) can be expressed as

$$
g_{q}=-\frac{1}{4} \sum_{i=1,2} \frac{a_{\widetilde{q}_{i}}^{2}+b_{\widetilde{q}_{i}}^{2}}{\left[m_{\widetilde{q_{i}}}^{2}-\left(m_{\widetilde{Z_{1}}}+m_{q}\right)^{2}\right]^{2}} .
$$

The gluon part of the scalar effective Lagrangian is fully determined by

$$
b=-T_{\widetilde{q}}+B_{D}+B_{S}-\frac{m_{\widetilde{Z}_{1}}}{2} B_{2 S}-\frac{m_{\widetilde{Z}_{1}}^{2}}{4}\left(B_{1 D}+B_{1 S}\right),
$$

where

$$
\begin{aligned}
T_{\widetilde{q}} & =\frac{1}{96 \pi} \sum_{j=1,2} \frac{c_{\widetilde{Z}}^{(j)}}{m_{H_{j}}^{2}} \sum_{q, i} \frac{c_{\widetilde{q_{i}}}^{(j)}}{m_{\widetilde{q}_{i}}^{2}} \\
B_{D} & =\frac{1}{32 \pi} \sum_{q, i}\left(a_{\widetilde{q_{i}}}^{2}-b_{\widetilde{q}_{i}}^{2}\right) m_{q} I_{1}\left(m_{\widetilde{q_{i}}}, m_{q}, m_{\widetilde{Z_{1}}}\right) \\
B_{S} & =\frac{1}{32 \pi} \sum_{q, i}\left(a_{\widetilde{q}_{i}}^{2}+b_{\widetilde{q}_{i}}^{2}\right) m_{\widetilde{Z}_{1}} I_{2}\left(m_{\widetilde{q_{i}}}, m_{q}, m_{\widetilde{Z_{1}}}\right) \\
B_{1 D} & =\frac{1}{12 \pi} \sum_{q, i}\left(a_{\widetilde{q}_{i}}^{2}-b_{\widetilde{q_{i}}}^{2}\right) m_{q} I_{3}\left(m_{\widetilde{q_{i}}}, m_{q}, m_{\widetilde{Z_{1}}}\right) \\
B_{1 S} & =\frac{1}{12 \pi} \sum_{q, i}\left(a_{\widetilde{q}_{i}}^{2}+b_{\widetilde{q_{i}}}^{2}\right) m_{\widetilde{Z}_{1}} I_{4}\left(m_{\widetilde{q_{i}}}, m_{q}, m_{\widetilde{Z_{1}}}\right) \\
B_{2 S} & =\frac{1}{48 \pi} \sum_{q, i}\left(a_{\widetilde{q}_{i}}^{2}+b_{\widetilde{q}_{i}}^{2}\right) I_{5}\left(m_{\widetilde{q_{i}}}, m_{q}, m_{\widetilde{Z_{1}}}\right) .
\end{aligned}
$$


The squark effective couplings in (B.16) are

$$
\begin{aligned}
c_{\widetilde{q_{1}}}^{(j)} & =\frac{g m_{Z}}{\cos \theta_{W}} s^{(j)}\left(T_{3 q} \cos ^{2} \theta_{q}-e_{q} \sin ^{2} \theta_{W} \cos 2 \theta_{q}\right) \\
& +\frac{g m_{q}^{2}}{m_{W}} r_{q}^{(j)}-\frac{g m_{q} \sin 2 \theta_{q}}{2 m_{W}}\left(\mu r_{q}^{\prime(j)}-A_{q} r_{q}^{(j)}\right), \\
c_{\widetilde{q}_{2}}^{(j)} & =\frac{g m_{Z}}{\cos \theta_{W}} s^{(j)}\left(T_{3 q} \sin ^{2} \theta_{q}+e_{q} \sin ^{2} \theta_{W} \cos 2 \theta_{q}\right) \\
& +\frac{g m_{q}^{2}}{m_{W}} r_{q}^{(j)}+\frac{g m_{q} \sin 2 \theta_{q}}{2 m_{W}}\left(\mu r_{q}^{\prime(j)}-A_{q} r_{q}^{(j)}\right),
\end{aligned}
$$

where

$$
s^{(1)}=-\cos (\alpha+\beta) s^{(2)}=\sin (\alpha+\beta)
$$

and

$$
\begin{gathered}
r_{u}^{\prime(1)}=-\frac{\cos \alpha}{\sin \beta}, \quad r_{u}^{\prime(2)}=\frac{\sin \alpha}{\sin \beta}, \\
r_{d}^{\prime(1)}=-\frac{\sin \alpha}{\cos \beta}, \quad r_{d}^{\prime(2)}=-\frac{\cos \alpha}{\cos \beta} .
\end{gathered}
$$

Loop integrals $I_{1}-I_{5}$ are given by Eqs. (B1a-e) in Ref. [8] (one must take care to correct the typo noted in Ref. [2]).

For the only effective coupling needed in the spin dependent Lagrangian we have

$$
d_{q}=\frac{1}{4} \sum_{i=1,2} \frac{a_{\widetilde{q}_{i}}+b_{\widetilde{q}_{i}}}{m_{\widetilde{q}_{i}}^{2}-\left(m_{\widetilde{Z}_{1}}+m_{q}\right)^{2}}-\frac{g^{2}}{4 m_{W}^{2}} O^{\prime \prime} T_{3 q},
$$

where

$$
O^{\prime \prime R}=\frac{1}{2}\left(N_{\ell 4}^{2}-N_{\ell 3}^{2}\right)
$$

is determined by the neutralino mass matrix diagonalizing matrix $N$. 


\section{REFERENCES}

[1] For reviews, see e.g. E. W. Kolb and M. S. Turner, The Early Universe, (AddisonWesley, Redwood City, 1989); J. Ellis, CERN-TH.7083/93 (1993); M. Drees, APCTP 96-04 (1996), hep-ph/9609300; see also Ref. [2].

[2] G. Jungman, M. Kamionkowski and K. Griest, Phys. Rep. 267, 195 (1996).

[3] H. Goldberg, Phys. Rev. Lett. 50, 1419 (1983); J. Ellis, J. Hagelin, D. Nanopoulos and M. Srednicki, Phys. Lett. B127, 233 (1983). Some recent papers include K. Griest, M. Kamionkowski and M. Turner, Phys. Rev. D41, 3565 (1990); P. Gondolo and G. Gelmini, Nucl. Phys. B360, 145 (1991); J. Ellis and L. Roszkowski, Phys. Lett. B283, 252 (1992); M. Drees and M. Nojiri, Phys. Rev. D47, 376 (1993); P. Nath and R. Arnowitt, Phys. Rev. Lett. 70, 3696 (1993); V. Berezinsky et. al., Astropart. Phys. 5, 1 (1996); V. Barger and C. Kao, MAD-PH-97-992 (1996); see also Ref. 田.

[4] H. Baer and M. Brhlik, Phys. Rev. D53, 597 (1996).

[5] For a review of dark matter detectors, see e.g. J. R. Primack, B. Sadoulet and D. Seckel, Ann. Rev. Nucl. Part. Sci. B38, 751 (1988); P. F. Smith and J. D. Lewin, Phys. Rep. 187, 203 (1990); D. Cline, UCLA-APH-0096-3-97 (1997).

[6] M. Goodman and E. Witten, Phys. Rev. D31, 3059 (1985)

[7] Some recent papers include K. Griest, Phys. Rev. D38, 2357 (1988), ibid. D39, 3802 (1989)(E) and Phys. Rev. Lett. 61, 666 (1988); M. Srednicki and R. Watkins, Phys. Lett. B225, 140 (1989); R. Barbieri, M. Frigeni and G. Giudice, Nucl. Phys. B313, 725 (1989); M. Kamionkowski, Phys. Rev. D44, 3021 (1991); J. Ellis and R. A. Flores, Phys. Lett. B263, 259 (1991); G. Gelmini, P. Gondolo and E. Roulet, Nucl. Phys. B351, 623 (1991); J. Engel, S. Pittel and P. Vogel, Int. J. Mod. Phys. E1, 1 (1992); T. Ressell et. al., Phys. Rev. D48, 5519 (1993); R. Arnowitt and P. Nath, Mod. Phys. Lett. A10, 1257 (1995), Phys. Rev. Lett. 74, 4592 (1995) and Phys. Rev. D54, 2374 (1996); E. Diehl, G. Kane, C. Kolda and J. Wells, Phys. Rev. D52, 4223 (1995); V. Bednyakov, H.V. Klapdor-Kleingrothaus and S. Kovalenko, Phys. Rev. D50, 7128 (1994); A. Bottino et. al., DFTT-75-96 (1996), hep-ph/9612451; see especially Ref. [8] and [2].

[8] M. Drees and M. Nojiri, Phys. Rev. D47, 4226 (1993) and Phys. Rev. D48, 3483 (1993).

[9] V. Dimitrov, J. Engel and S. Pittel, Phys. Rev. D51, 291 (1995).

[10] A. Chamseddine, R. Arnowitt and P. Nath, Phys. Rev. Lett. 49, 970 (1982); R. Barbieri, S. Ferrara and C. Savoy, Phys. Lett. B119, 343 (1982); L.J. Hall, J. Lykken and S. Weinberg, Phys. Rev. D27, 2359 (1983); for recent reviews, see M. Drees and S. Martin in Electroweak Symmetry Breaking and New Physics at the TeV Scale, edited by T. Barklow, S. Dawson, H . Haber and J. Seigrist, (World Scientific) 1995, hep-ph/9504324 and X. Tata, UH-511-872-97 (1997), hep-ph/9706307.

[11] F. Paige and S. Protopopescu, in Supercollider Physics, p. 41, ed. D. Soper (World Scientific, 1986); H. Baer, F. Paige, S. Protopopescu and X. Tata, in Proceedings of the Workshop on Physics at Current Accelerators and Supercolliders, ed. J. Hewett, A. White and D. Zeppenfeld, (Argonne National Laboratory, 1993), hep-ph/9305342.

[12] R. A. Flores, Phys. Lett. B215, 73 (1988); see also M. S. Turner, Phys. Rev. D33, 899 (1986) and E. Gates, G. Gyuk and M. Turner, FERMILAB-Pub-95/090-A (1995), astro-ph/9505039.

[13] H. L. Lai et. al., Phys. Rev. D51, 4763 (1995).

[14] J. Engel, Phys. Lett. B264, 114 (1991). 
[15] J. Ashman et. al. (EMC Collaboration), Phys. Lett. B206, 364 (1988); R. L. Jaffe and A. Manohar, Nucl. Phys. B337, 509 (1990).

[16] V. Dimitrov, J. Engel and S. Pittel, Phys. Rev. D51, 291 (1995).

[17] Y. Pan, presented at PHENO 9\%: Recent Developments in Phenomenology, Madison, WI, March 1997.

[18] H. Baer, C. H. Chen, M. Drees, F. Paige and X. Tata, FSU-HEP-970501 (1997), hepph/9704457.

[19] See for example S. Bertolini, F. Borzumati, A. Masiero and G. Ridolfi, Nucl. Phys. B353, 591 (1991); R. Barbieri and G. F. Giudice, Phys. Lett. B309, 86 (1993); F. Borzumati, M. Drees and M. Nojiri, Phys. Rev. D51, 341 (1995); V. Barger, M. Berger, P. Ohmann and R. Phillips, Phys. Rev. D51, 2438 (1995); J. Lopez, D. Nanopoulos, X. Wang and A. Zichichi, Phys. Rev. D51, 147 (1995); J. Wu, R. Arnowitt and P. Nath, Phys. Rev. D51, 1371 (1995); B. de Carlos and J. A. Casas, Phys. Lett. B349, 300 (1995) and ERRATUM-ibid B351, 604 (1995).

[20] H. Baer and M. Brhlik, Phys. Rev. D55, 3201 (1997).

[21] H. Baer, M. Brhlik, R. Munroe and X. Tata, Phys. Rev. D52, 5031 (1995).

[22] H. Baer, C. H. Chen, C. Kao and X. Tata, Phys. Rev. D52, 1565 (1995); H. Baer, C. H. Chen, F. Paige and X. Tata, Phys. Rev. D54, 5866 (1996).

[23] H. Baer, C. H. Chen, F. Paige and X. Tata, Phys. Rev. D52, 2746 (1995) and Phys. Rev. D53, 6241 (1996). 
FIGURES
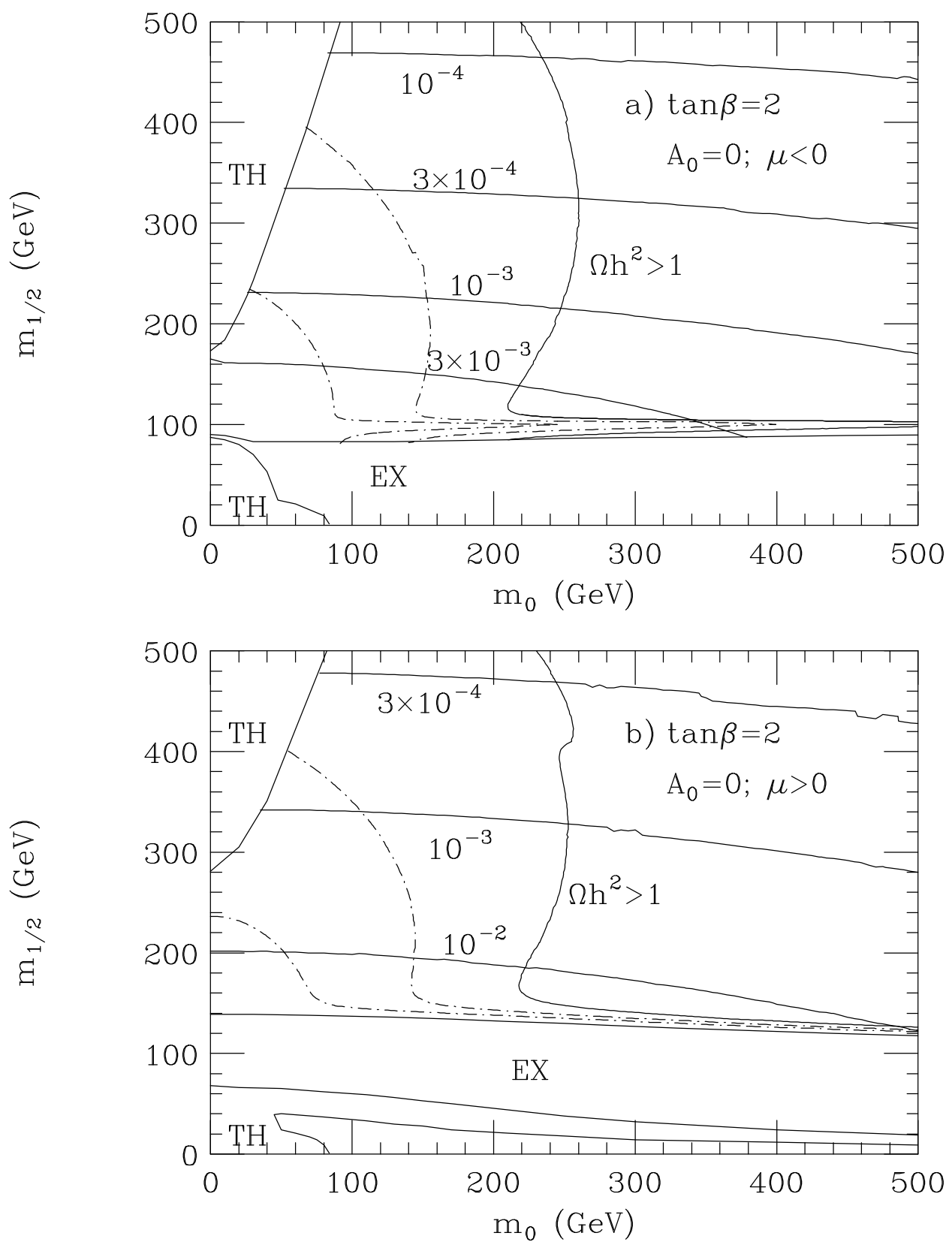

FIG. 1. A plot of contours of neutralino scattering events $/ \mathrm{kg} /$ day in a ${ }^{73} \mathrm{Ge}$ detector, for mSUGRA parameters $A_{0}=0, \tan \beta=2$ and $\left.a\right) \mu<0$ and $\left.b\right) \mu>0$, in the $m_{0}$ vs. $m_{1 / 2}$ plane. The regions labelled TH are excluded by theoretical considerations, while the EX regions are excluded by collider searches for SUSY particles. The region to the right of the solid contour is excluded by $\Omega h^{2}>1$. We also show contours of neutralino relic density $\Omega h^{2}=0.15$ and 0.4 ; the region in between is favored by models of a MDM universe. 

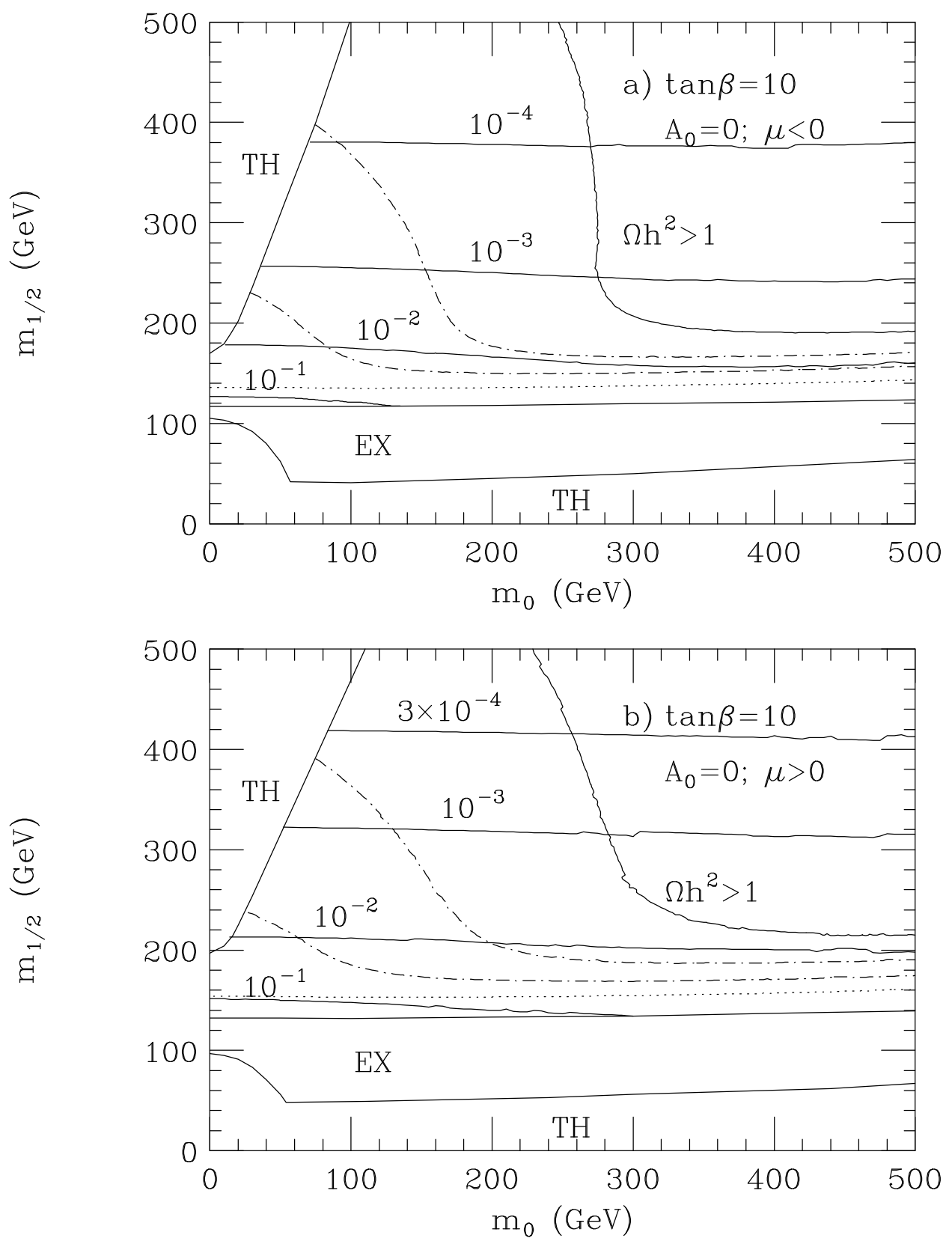

FIG. 2. Same as Fig. 1, except for $\tan \beta=10$. Below the dotted contour is where $\Omega h^{2}<0.025$. 

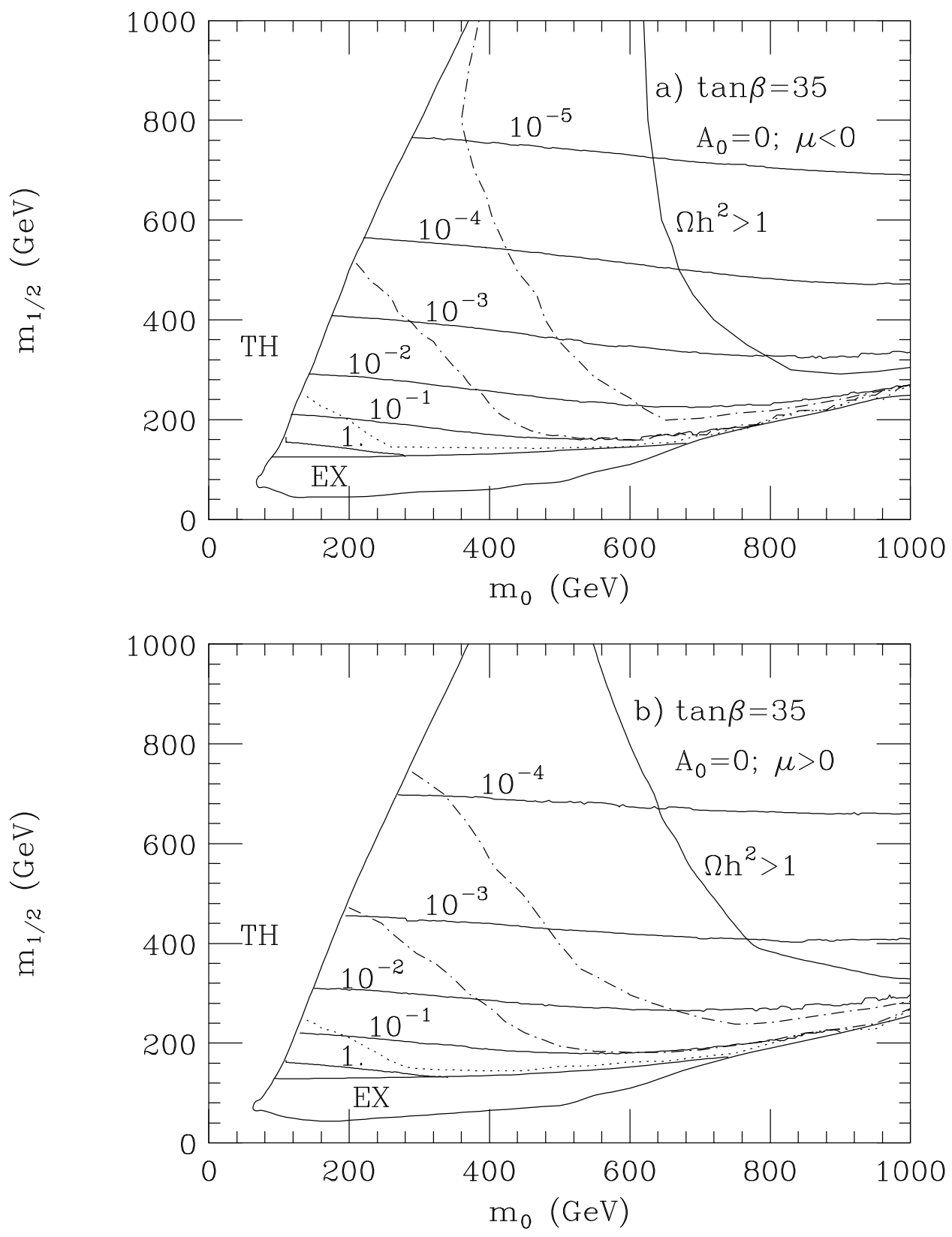

FIG. 3. Same as Fig. 1, except for $\tan \beta=35$. Note, however, the expanded scale relative to Fig. 1 and 2. 


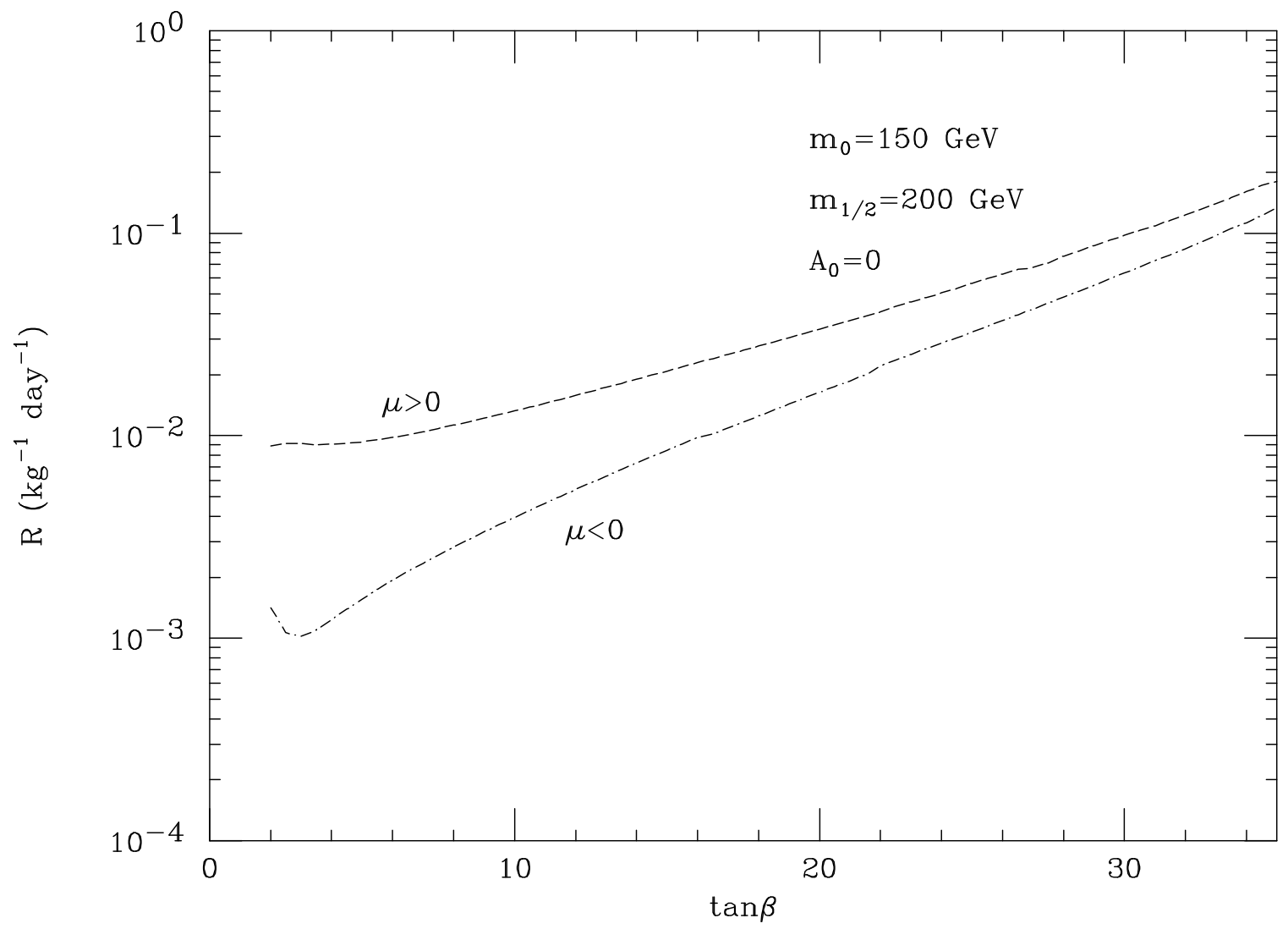

FIG. 4. A plot of neutralino scattering events/kg/day in a ${ }^{73}$ Ge detector, for mSUGRA parameters $m_{0}, m_{1 / 2}, A_{0}=150,200$, and $0 \mathrm{GeV}$ versus $\tan \beta$ for $\mu<0$ and $\mu>0$. 


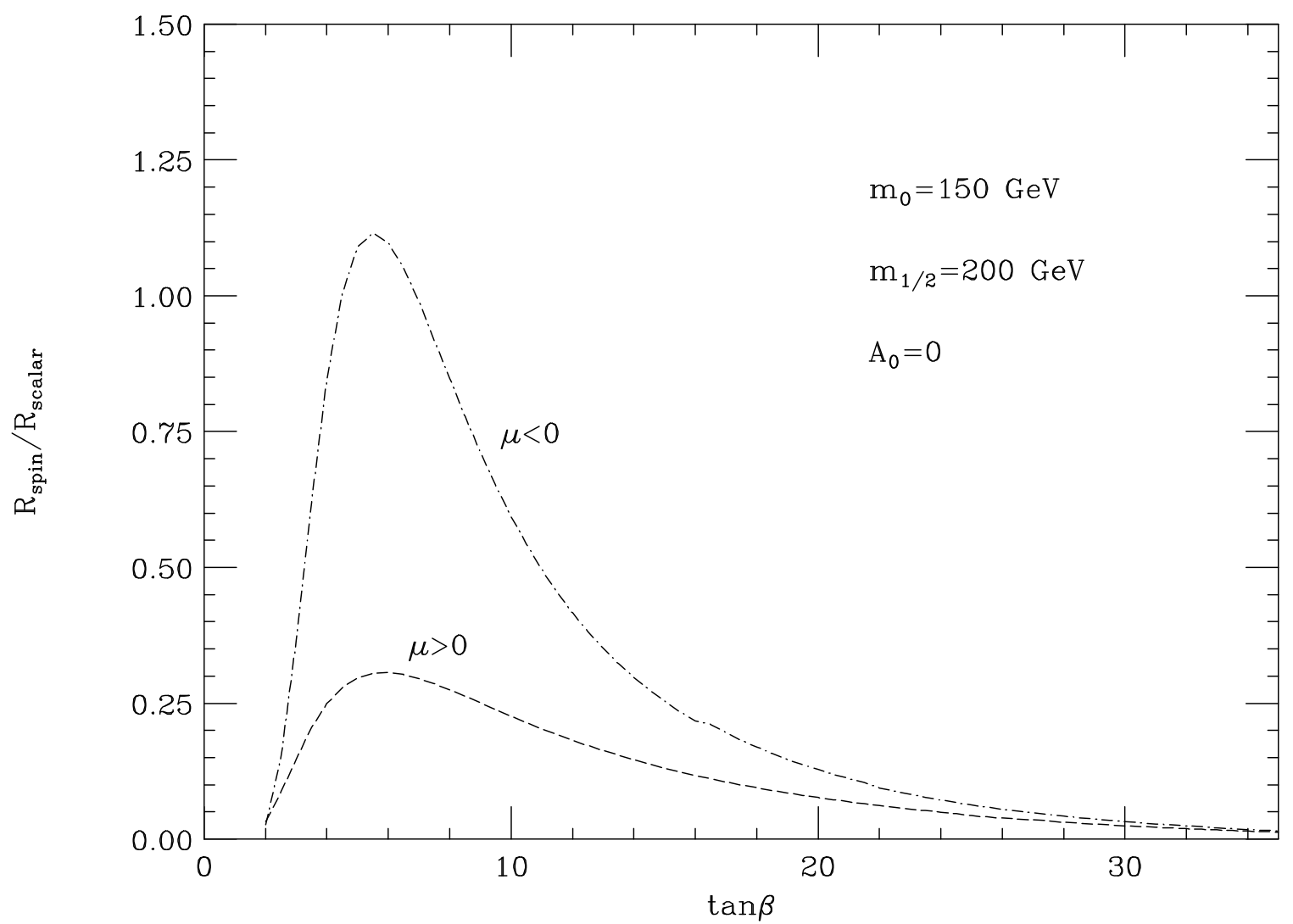

FIG. 5. A plot of the ratio of neutralino scattering events from the spin-spin interaction over that from scalar interactions for mSUGRA parameters $m_{0}, m_{1 / 2}, A_{0}=150,200$, and $0 \mathrm{GeV}$, versus $\tan \beta$ for $\mu<0$ and $\mu>0$. 


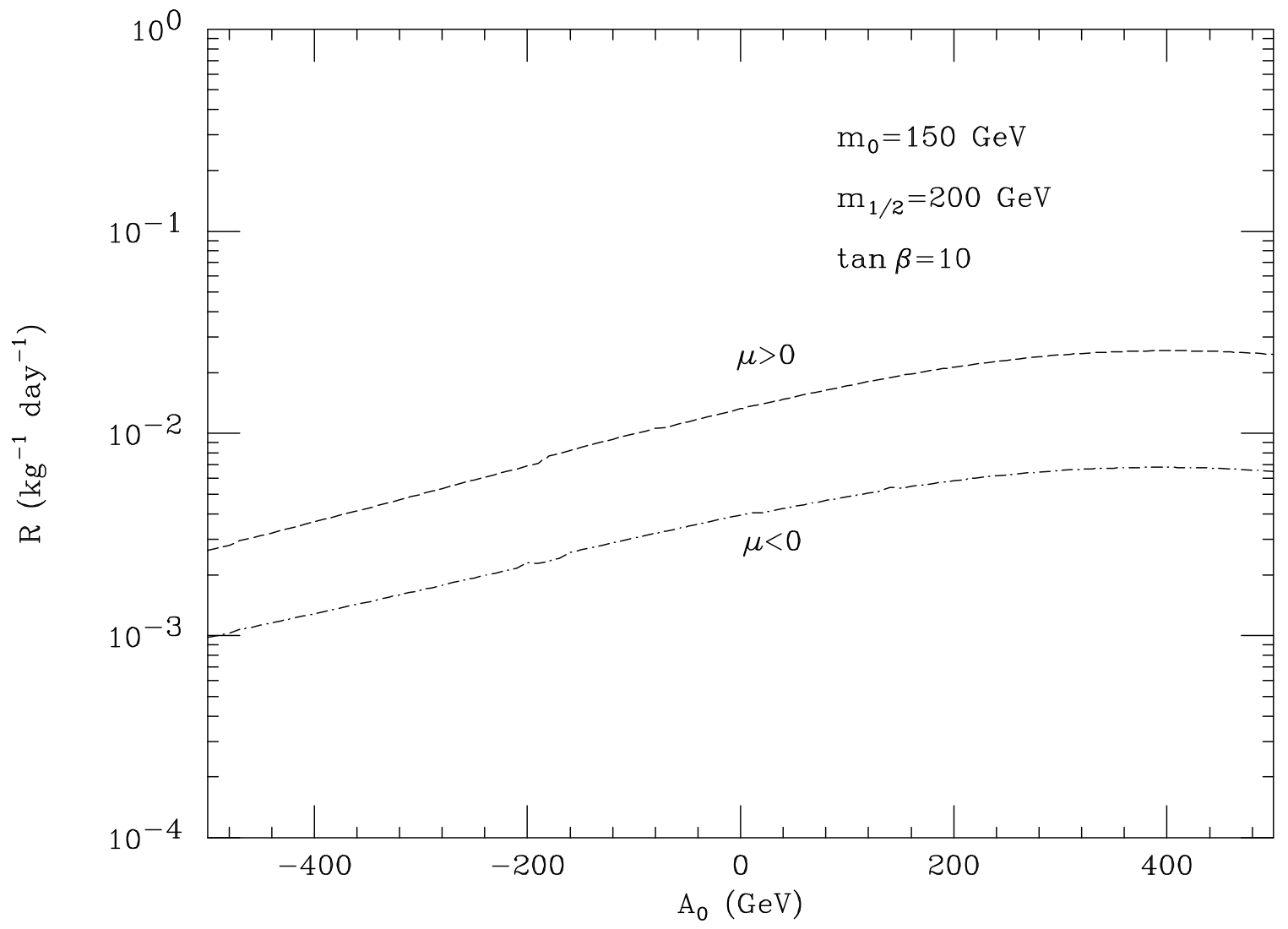

FIG. 6. A plot of neutralino scattering events $/ \mathrm{kg} /$ day in a ${ }^{73} \mathrm{Ge}$ detector, for mSUGRA parameters $m_{0}, m_{1 / 2}=150,200 \mathrm{GeV}$ with $\tan \beta=10$, versus the parameter $A_{0}$, for $\mu<0$ and $\mu>0$. 


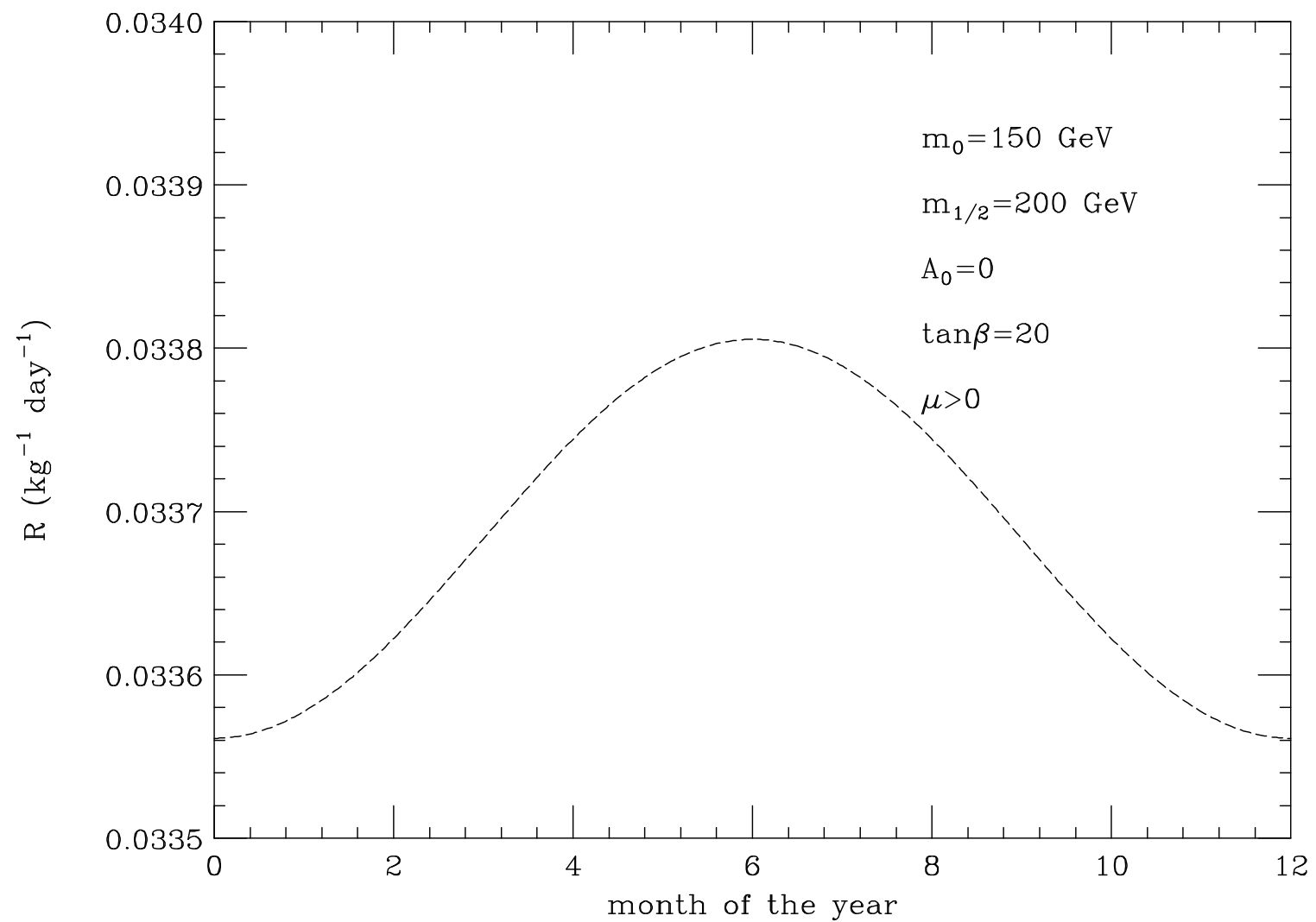

FIG. 7. A plot of neutralino scattering events $/ \mathrm{kg} /$ day in a ${ }^{73} \mathrm{Ge}$ detector for mSUGRA parameters $m_{0}, m_{1 / 2}, A_{0}=150,200,0 \mathrm{GeV}$ with $\tan \beta=20$ and $\mu>0$, versus month of the year, from Jan. 1 to Dec. 31, showing the expected seasonal variation in dark matter detection rate. 

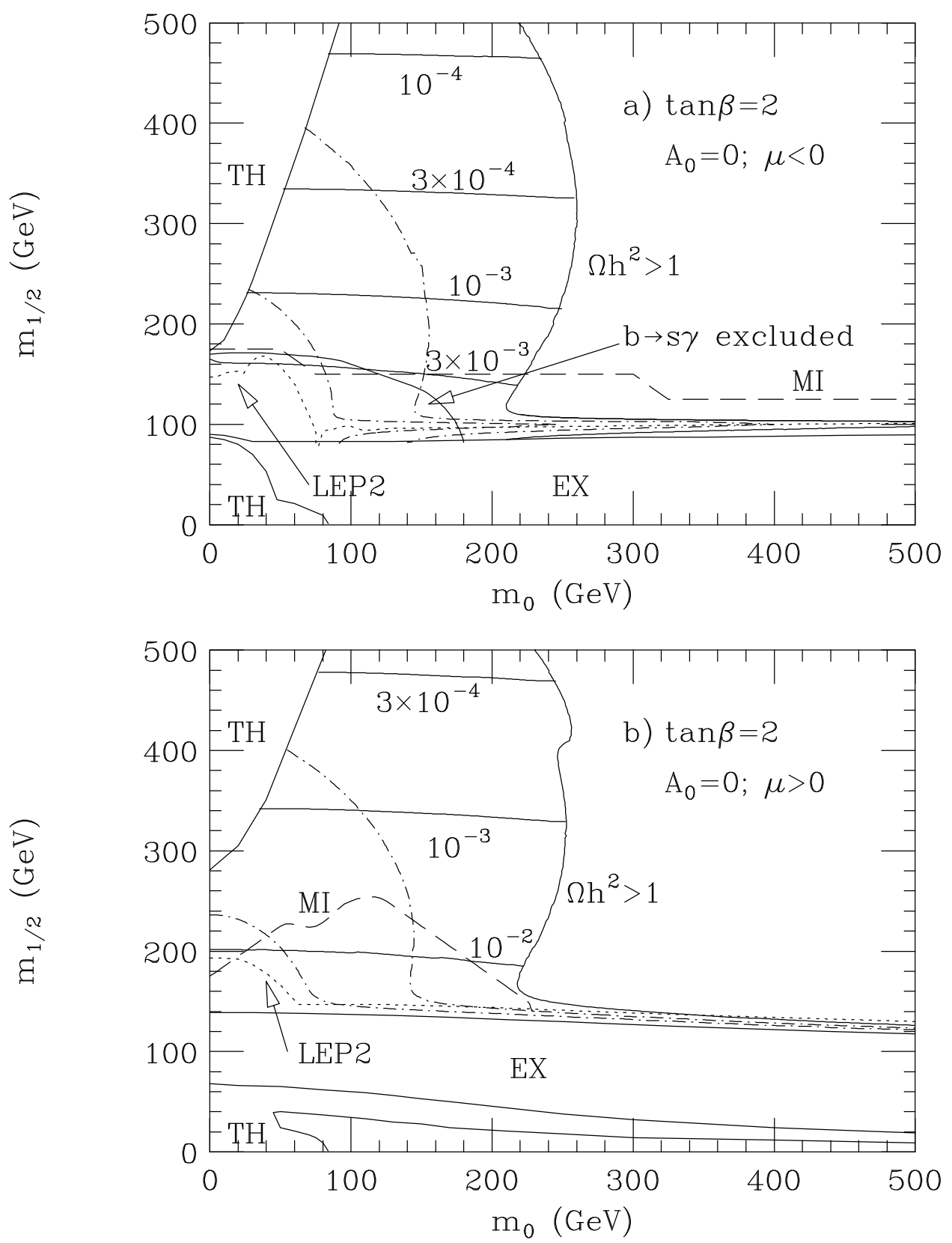

FIG. 8. Same as Fig. 1, but with in addition $b \rightarrow s \gamma$ exclusion contours, plus the reach contours for LEP2 and Fermilab Tevatron MI experiments for detecting SUSY. 

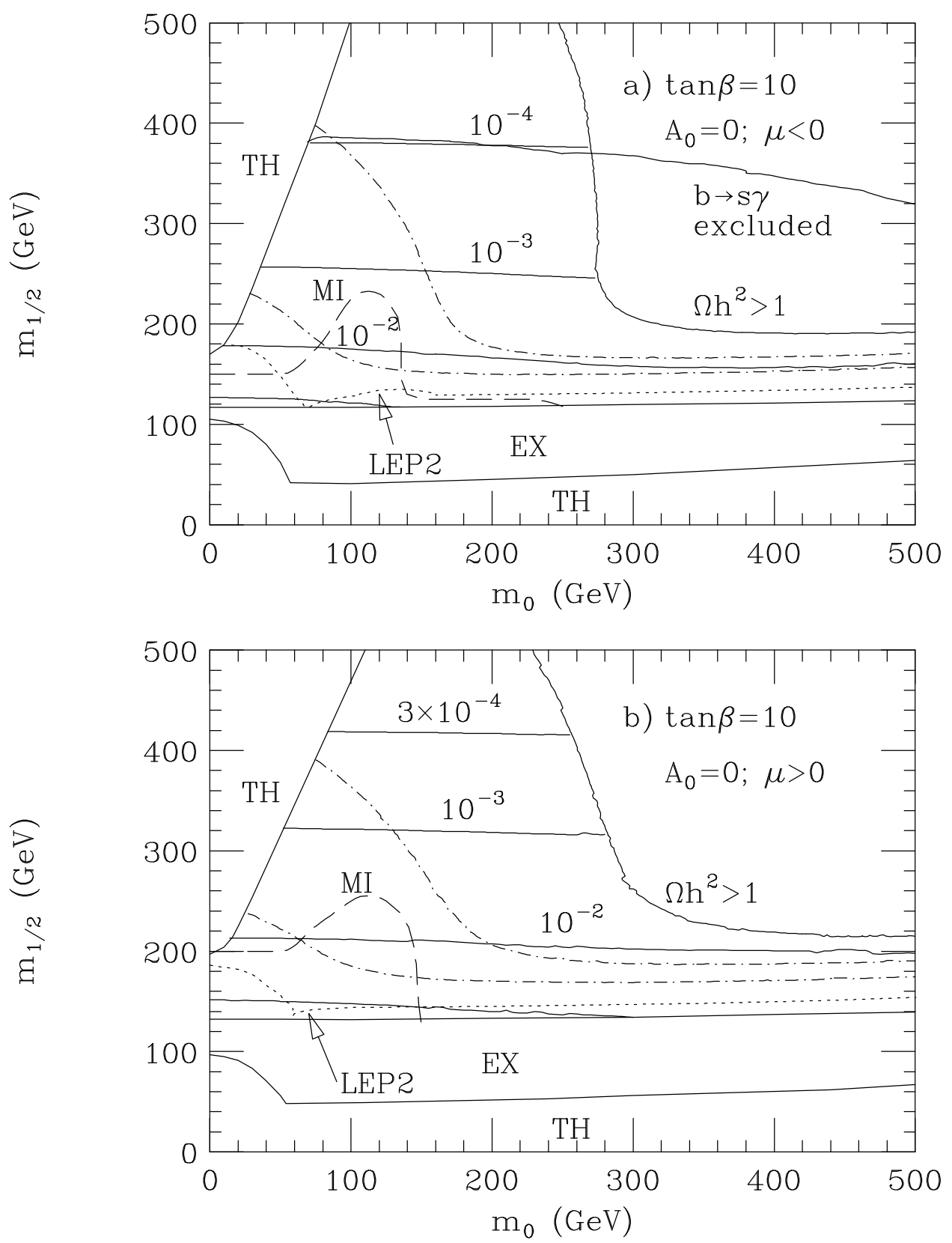

FIG. 9. Same as Fig. 2, but with in addition $b \rightarrow s \gamma$ exclusion contours, plus the reach contours for LEP2 and Fermilab Tevatron MI experiments for detecting SUSY. 